\title{
STATE FOR LINEAR TIME-VARYING SYSTEMS, WITH APPLICATIONS TO DISSIPATIVE SYSTEMS
}

\author{
P. RAPISARDA*
}

\begin{abstract}
An intrinsic definition of state is given for systems described by higher-order linear differential equations with time-varying coefficients. Based on this definition we characterize a polynomial differential operator that acting on a system trajectory defines a corresponding state one, and we illustrate a procedure to compute a state variable from the differential equations. We prove that there exist representations of first order in such state variable and zeroth order in the input and output variables. We also consider linear, time-varying dissipative systems, and we give several characterisations of the property of cyclo-losslessness. We prove that for dissipative systems the storage function is a quadratic function of the state.
\end{abstract}

Key words. State variable, differential-algebraic equations with time-varying coefficients, dissipativity, storage function, bilinear and quadratic differential forms.

AMS subject classifications. 93A10, 93A30, 93B25, 93C05

1. Introduction. We consider systems described by higher-order linear differential equations with time-varying coefficients, called linear time-varying differential systems in the following. These are the natural end-product of a modelling procedure based on tearing a complex system in subsystems, zooming in each of them to model it based on first principles, and combining the sub-models in a global mathematical description (see [21]). In our setting, state variables are auxiliary ones computed from the higher-order representation, and not given a priori. The characteristic property of state is that it "splits" past and future: its value at a time instant $t_{0}$ determines whether the concatenation of two system trajectories at $t_{0}$ is itself an admissible system trajectory. In this paper, we show how a state variable can be computed from the system equations using this definition, and we show how such variables arise when considering energy supply and storage functionals.

We studied state construction for higher-order linear differential equations with constant coefficients in $[15,17]$; the present work differs from it in many respects. Firstly, the analysis in $[15,17]$ heavily relied on the algebra of polynomial matrices, but here we work directly with linear differential operators with time-varying coefficients. Secondly, the technical issues involved are considerably different; fortunately, previous work (most notably [8, 9, 23, 24]) was of great help in reducing such difficulties to a complexity manageable by this author. Thirdly, we extend our analysis to the study of bilinear and quadratic functionals of linear time-varying differential systems. We work directly with such functionals, without relying on the algebra of bi-variate polynomials as done in [20] for the linear time-invariant case. We prove that storage functions are quadratic functions of the state also for the time-varying case.

The literature on linear time-varying systems is vast, but only some authors have taken higher-order differential equations as a starting point (see [7, 8, 9, 23]), and even fewer have considered the state realization problem in this framework (see [4, 11, 24]). We briefly mention their contributions here, deferring to remarks interspersed in the text more specific analyses of their relation with the results presented in this paper.

A common feature of past contributions is that the state realization problem is studied not from a trajectory-based point of view, with the state property occupying a central role as it does in this paper, but rather as the classical problem of devising

*Vision, Learning and Control group, University of Southampton, UK, (pr3@ecs.soton.ac.uk). 
auxiliary variables with respect to which first-order representations can be computed. Only in sect. V.A of [24] does the state property appear, but as a consequence of the existence of first order representations. The authors of [4] consider higherorder differential equations with smooth time-varying coefficients, and compute a minimal state representation for the single-input, single-output case using algebraic manipulations of the differential equation. In [11], the authors use the algebra of noncommutative polynomial rings to construct a state variable for the multiple-input, multiple-output case. Their elegant approach connecting polynomial division and state construction has the potential of bringing computer algebra techniques to bear on the realisation problem. Our results about quadratic and bilinear forms in the time-varying case are completely original in subject and technique.

Throughout the paper we make extensive use of behavioral system theory concepts; the reader is referred to $[19,21]$ for an introduction. In sect. 2 we define the solution space, system representations, and the concept of state. In sect. 3 we characterize concatenability of system trajectories and we compute a state variable. We consider system representations with auxiliary variables in sect. 4 . In sect. 5 we prove that the state variable defined in sect. 3 induces first order system representations. In sect. 6 we give several characterizations of losslessness and we prove that storage functions are quadratic functions of the state. Sect. 7 concludes the paper.

2. Basic definitions. We consider a system to be essentially characterized by its behavior, i.e. the set of trajectories satisfying a system of linear differential equations with time-varying coefficients. These equations may involve only the variables one is interested in modelling, for example the voltage and current at the external ports of an electrical circuit; these are called external variables and are denoted by $w$ in the following. A system representation involving only the external variables is

$$
R_{0} w(\cdot)+\ldots+R_{L} \frac{d^{L}}{d t^{L}} w(\cdot)=0,
$$

where $R_{k}$ is a $p \times q$ matrix whose entries are meromorphic functions on $\mathbb{R}, k=0, \ldots, L$, $w$ is the $q$-dimensional vector of external variables, and $w(\cdot)$ denotes a trajectory in the variables $w$. In the following we write (2.1) compactly as $R\left(\frac{d}{d t}\right) w(\cdot)=0$, where $R\left(\frac{d}{d t}\right)$ is the polynomial differential operator with meromorphic coefficients defined by $R\left(\frac{d}{d t}\right):=R_{0}+\ldots+R_{L} \frac{d^{L}}{d t^{L}}$. This justifies the terminology kernel representation for the set of solutions of $(2.1)$.

In $[3,9,22,23]$ various concepts of solution for $(2.1)$ have been discussed. In this paper we follow $[9,23]$ and opt for piecewise smooth functions, i.e. the space consisting of all functions $w(\cdot)$ of one real argument and taking values in $\mathbb{R}^{q}$ for which there exists a discrete set $E(w) \subset \mathbb{R}$ such that $w(\cdot) \in \mathcal{C}^{\infty}\left(\mathbb{R} \backslash E(w), \mathbb{R}^{q}\right)$. We denote the set of such functions by the symbol $\mathcal{C}_{\mathrm{pw}}^{\infty}\left(\mathbb{R}, \mathbb{R}^{q}\right)$. The reader is referred to sect. 1.3 of [9] for examples of the subtleties involved in defining the set of solutions of differential equations with time-varying coefficients, and why $\mathcal{C}_{\mathrm{pw}}^{\infty}\left(\mathbb{R}, \mathbb{R}^{q}\right)$ is a suitable choice for engineering purposes. A solution may not be defined everywhere (see sect. 1.3 of [9]), and the notation $\operatorname{dom}(w) \subseteq \mathbb{R}$ is used in the following to denote the domain of $w(\cdot) \in \mathcal{C}_{\mathrm{pw}}^{\infty}\left(\mathbb{R}, \mathbb{R}^{q}\right)$.

The set of solutions to (2.1) is called the (global) behavior defined by

$$
\begin{aligned}
\mathcal{B} & :=\left\{w(\cdot) \in \mathcal{C}_{\mathrm{pw}}^{\infty}\left(\mathbb{R}, \mathbb{R}^{q}\right) \mid R\left(\frac{d}{d \tau}\right) w(\tau)=0 \text { for almost all } \tau\right\} \\
& =: \operatorname{ker} R\left(\frac{d}{d t}\right) .
\end{aligned}
$$


In the rest of this paper we denote the set of meromorphic functions from $\mathbb{R}$ to $\mathbb{R}$ by $\mathcal{M}$, and the set of $p \times q$ matrices with meromorphic entries by $\mathcal{M}^{p \times q}$.

Sometimes it is necessary for modelling purposes to introduce auxiliary variables besides the external ones; for example, when modelling electrical circuits, voltages and currents in the internal branches are needed to model the port variables. System representations involving the auxiliary variables (denoted by $\ell$ in the following, and equivalently called "latent" in the following) besides the external ones $w$ are of the form

$$
R_{0} w(\cdot)+\ldots+R_{L} \frac{d^{L}}{d t^{L}} w(\cdot)=M_{0} \ell(\cdot)+M_{N} \frac{d^{N}}{d t^{N}} \ell(\cdot),
$$

where $R_{k} \in \mathcal{M}^{g \times q}, k=0, \ldots, L, M_{j} \in \mathcal{M}^{g \times m}, j=0, \ldots, N$, and $w(\cdot) \in \mathcal{C}_{\mathrm{pw}}^{\infty}\left(\mathbb{R}, \mathbb{R}^{q}\right)$, $\ell(\cdot) \in \mathcal{C}_{\mathrm{pw}}^{\infty}\left(\mathbb{R}, \mathbb{R}^{m}\right)$. Using standard terminology in behavioral system theory (see e.g. [19]), we call (2.3) a hybrid representation (since it involves both external and auxiliary variables). The equations (2.3) define the (global) full behavior

$$
\mathcal{B}_{f}:=\left\{(w(\cdot), \ell(\cdot)) \in \mathcal{C}_{\mathrm{pw}}^{\infty}\left(\mathbb{R}, \mathbb{R}^{q+m}\right) \mid(2.3) \text { holds almost everywhere (a.e.) }\right\}
$$

and the (global) external behavior

$$
\mathcal{B}_{e}:=\left\{w(\cdot) \in \mathcal{C}_{\mathrm{pw}}^{\infty}\left(\mathbb{R}, \mathbb{R}^{q}\right) \mid \exists \ell(\cdot) \in \mathcal{C}_{\mathrm{pw}}^{\infty}\left(\mathbb{R}, \mathbb{R}^{m}\right) \text { s.t. (2.3) holds a.e. }\right\}
$$

In the case of electrical circuits, the full behavior consists of the trajectories of the internal currents and voltages and of those at the ports, while the external behavior consists of only the trajectories of the port variables.

A special case of the representation (2.3) occurs when $R\left(\frac{d}{d t}\right)=I_{q}$; in this case

$$
w(\cdot)=M_{0} \ell(\cdot)+\ldots+M_{N} \frac{d^{N}}{d t^{N}} \ell(\cdot),
$$

is called an image representation. A linear time-varying system is representable in image form if and only if it is controllable. (For the definition of controllability, see Def. 3.1 p. 1734 of [9] or Def. 3 p. 122 of [23]; and for the equivalence of controllability and representability in image form, see Th. 7 p. 122 of [23].)

In the following the set of solutions of a system of differential equations with time-varying coefficients is called a linear time-varying differential behavior. We refer the reader to sect. 6 of [9] and sect. 5.3 of [23] for a thorough treatment of how hybrid and kernel representations are related to each other via the "elimination of latent variables" theorem.

We now introduce a trajectory-based point of view on the concept of state. In our definition of state we use the concatenation of two trajectories $f_{k}$ at $t_{0}, k=1,2$, denoted by the symbol $f_{1}(\cdot) \underset{t_{0}}{\wedge} f_{2}(\cdot)$, whose value at $t$ is defined by

$$
\left(f_{1} \hat{t}_{0} f_{2}\right)(t):=\left\{\begin{array}{ll}
f_{1}(t) & \text { if } t<t_{0}, t \in \operatorname{dom}\left(f_{1}\right) \\
f_{2}(t) & \text { if } t \geq t_{0}, t \in \operatorname{dom}\left(f_{2}\right)
\end{array} .\right.
$$

State variables are a special kind of auxiliary variables associated with the property of concatenability between full (internal and external) trajectories (see also [15]). 
DEFINITION 2.1. Let $\mathcal{B}_{f}$ be a full behavior with external variables $w$ and auxiliary variables $\ell$. The variables $\ell$ are $a$ state for $\mathcal{B}_{f}$ if for all $\left(w_{1}(\cdot), \ell_{1}(\cdot)\right),\left(w_{2}(\cdot), \ell_{2}(\cdot)\right) \in \mathcal{B}_{f}$ and all $t_{0} \in \operatorname{dom}\left(w_{1}(\cdot), \ell_{1}(\cdot)\right) \cap \operatorname{dom}\left(w_{2}(\cdot), \ell_{2}(\cdot)\right)$ it holds that

$(2.8)\left[\ell_{1}(\cdot) \underset{t_{0}}{\wedge} \ell_{2}(\cdot)\right.$ continuous at $\left.t_{0}\right] \Longrightarrow\left[\left(w_{1}(\cdot), \ell_{1}(\cdot)\right) \underset{t_{0}}{\wedge}\left(w_{2}(\cdot), \ell_{2}(\cdot)\right) \in \mathcal{B}_{f}\right]$.

If $\ell$ is a state variable, $\mathcal{B}_{f}$ is called a state system for $\mathcal{B}_{e}$ defined by (2.5).

REMARK 1. Def. 2.1 is slightly different from the corresponding one for timeinvariant systems, see formula (3.3) p. 1058 in [15]. Since we work with piecewise smooth functions with only a discrete set of discontinuities, we identify functions that coincide outside a discrete set. Consequently, we only require continuity at $t=t_{0}$, instead of pointwise equality at $t=t_{0}$ (as in [15]). On this issue, see also the definition of equivalence in formula (2.5) p. 2418 of [17].

In the next two sections we consider the characterization of state variables for systems described by (2.1) and (2.6).

3. State from external variables: kernel representations. We first characterize concatenability of two external trajectories of (2.1) as conditions on the trajectories and their derivatives at the concatenation instant. Linearity implies that we can reduce ourselves to the case when one of the two trajectories is identically zero.

Proposition 3.1. Let $\mathcal{B}$ be a linear time-varying behavior, and let $w(\cdot), w^{\prime}(\cdot) \in \mathcal{B}$ and $t_{0} \in \mathbb{R}$. Then $\left(w(\cdot) \underset{t_{0}}{\wedge} w^{\prime}(\cdot)\right) \in \mathcal{B}$ if and only if $\left(0 \hat{t}_{0} w^{\prime}(\cdot)-w(\cdot)\right) \in \mathcal{B}$.

Proof. Straightforward from linearity.

Using the equivalence stated in Prop. 3.1, we study the conditions under which a trajectory is concatenable with zero.

Let $\mathbb{I}:=[a, b] \subset \mathbb{R}$ be a fixed interval. On $\mathcal{C}^{\infty}\left(\mathbb{I}, \mathbb{R}^{q}\right)$ the differential operator $P \frac{d^{j}}{d t^{j}}$ with $P \in \mathcal{M}^{m \times n}$ has a (unique) formal adjoint defined by

$$
\left(P \frac{d^{j}}{d t^{j}}\right)^{*}:=(-1)^{j}\left(P^{\top} \circ i d\right)^{(j)}=(-1)^{j} \sum_{i=0}^{j}\left(\begin{array}{l}
j \\
i
\end{array}\right) P^{(i) \top} \frac{d^{j-i}}{d t^{j-i}},
$$

see e.g. Th. 3.1 p. 303 of [12]. It follows that every polynomial differential operator $R\left(\frac{d}{d t}\right)$ with $m \times n$ meromorphic coefficients defined on $\mathbb{I}$ has an adjoint operator, denoted by $R\left(\frac{d}{d t}\right)^{*}$, such that for every $f \in \mathcal{C}^{\infty}\left(\mathbb{I}, \mathbb{R}^{n}\right), g \in \mathcal{C}^{\infty}\left(\mathbb{I}, \mathbb{R}^{m}\right)$ with zero boundary conditions, the equality

$$
\int_{a}^{b} f^{\top}\left(R\left(\frac{d}{d t}\right) g\right) d t=\int_{a}^{b}\left(R\left(\frac{d}{d t}\right)^{*} f\right)^{\top} g d t
$$

holds. A closed form expression for $R\left(\frac{d}{d t}\right)^{*}$ follows from (3.1):

$$
R\left(\frac{d}{d t}\right)^{*}=\left(R_{0}+\ldots+R_{L} \frac{d^{L}}{d t^{L}}\right)^{*}=\sum_{j=0}^{L}(-1)^{j} \sum_{i=0}^{j}\left(\begin{array}{l}
j \\
i
\end{array}\right) R_{j}^{(i) \top} \frac{d^{j-i}}{d t^{j-i}} .
$$

We now characterize concatenability with zero at $t_{0}$. 
$182 \int_{t_{0}}^{b} f^{\top}\left[R_{0} w+\left(\sum_{k=1}^{L} R_{k} w^{(k)}\right)\right] d t$

$183=\int_{t_{0}}^{b}\left[f^{\top} R_{0} w+\sum_{k=1}^{L} \frac{d}{d t}\left(\sum_{j=0}^{k-1}(-1)^{j}\left(f^{\top} R_{k}\right)^{(j)} w^{(k-1-j)}\right)+(-1)^{k}\left(f^{\top} R_{k}\right)^{(k)} w\right] d t$,

TheOREm 3.2. Let (2.1) be a kernel representation of $\mathcal{B}$. Define the polynomial differential operator $X_{m}\left(\frac{d}{d t}\right), m=0, \ldots, L-1$ by

$$
X_{m}\left(\frac{d}{d t}\right) w(\cdot):=\sum_{k=m+1}^{L} \sum_{j=m}^{k-1}(-1)^{j}\left(\begin{array}{c}
j \\
j-m
\end{array}\right) R_{k}^{(j-m)} w^{(k-1-j)}(\cdot) .
$$

Let $w(\cdot) \in \mathcal{B}$; the following equivalence holds:

$$
\left(0 \wedge \hat{t_{0}}\right)(\cdot) \in \mathcal{B} \Longleftrightarrow X_{m}\left(\frac{d}{d t}\right) w\left(t_{0}\right)=0, m=0, \ldots, L-1
$$

Proof. Since the set of singularities of the coefficients $R_{k}, k=0, \ldots, L$ is discrete, there exists an interval $\mathbb{I}\left(t_{0}\right):=[a, b]$ containing $t_{0}$ on which $R_{k}, k=0, \ldots, L$ are all defined. In the following, we denote by $\mathcal{F}_{0}\left(\mathbb{I}\left(t_{0}\right)\right)$ the set of all smooth functions which are identically zero in a neighborhood of the extremes of the interval $\mathbb{I}\left(t_{0}\right)$. The equality $\int_{a}^{b} f(\cdot)^{\top}\left(R\left(\frac{d}{d t}\right) w(\cdot)\right) d t=0$ holds for all $f(\cdot) \in \mathcal{F}_{0}\left(\mathbb{I}\left(t_{0}\right)\right)$ if and only if $\int_{a}^{b}\left(R\left(\frac{d}{d t}\right)^{*} f(\cdot)\right)^{\top} w(\cdot) d t=0$ for all $f(\cdot) \in \mathcal{F}_{0}\left(\mathbb{I}\left(t_{0}\right)\right)$. Note that $\left(0 \wedge \underset{t_{0}}{\wedge}\right)(\cdot) \in \mathcal{B}$ if and only if for every $f(\cdot) \in \mathcal{F}_{0}\left(\mathbb{I}\left(t_{0}\right)\right)$ it holds that

$$
\begin{aligned}
0 & =\int_{a}^{b} f^{\top}\left(\sum_{k=0}^{L} R_{k}\left(0 \underset{t_{0}}{\wedge} w\right)^{(k)}\right) d t=\int_{t_{0}}^{b} f^{\top}\left(\sum_{k=0}^{L} R_{k} w^{(k)}\right) d t \\
& =\int_{t_{0}}^{b} f^{\top}\left[R_{0} w+\left(\sum_{k=1}^{L} R_{k} w^{(k)}\right)\right] d t .
\end{aligned}
$$

We now prove that for every $k \geq 1$

$$
f^{\top}\left(R_{k} w^{(k)}\right)=\frac{d}{d t}\left[\sum_{j=0}^{k-1}(-1)^{j}\left(f^{\top} R_{k}\right)^{(j)} w^{(k-1-j)}\right]+(-1)^{k}\left(f^{\top} R_{k}\right)^{(k)} w .
$$

This follows from $(-1)^{k-1}+(-1)^{k}=0$ and

$$
\begin{aligned}
\frac{d}{d t}\left[\sum_{j=0}^{k-1}(-1)^{j}\left(f^{\top} R_{k}\right)^{(j)} w^{(k-1-j)}\right] & =(-1)^{0}\left[\left(f^{\top} R_{k}\right)^{(1)} w^{(k-1)}+\left(f^{\top} R_{k}\right)^{(0)} w^{(k)}\right] \\
& +(-1)^{1}\left[\left(f^{\top} R_{k}\right)^{(2)} w^{(k-2)}+\left(f^{\top} R_{k}\right)^{(1)} w^{(k-1)}\right]+\ldots \\
& =(-1)^{0}\left(f^{\top} R_{k}\right)^{(0)} w^{(k)}+(-1)^{k-1}\left(f^{\top} R_{k}\right)^{(k)} w^{(0)}
\end{aligned}
$$

Use equation (3.6) to rewrite $\int_{t_{0}}^{b} f^{\top}\left[R_{0} w+\left(\sum_{k=1}^{L} R_{k} w^{(k)}\right)\right] d t$ as 
and conclude that

$$
\begin{aligned}
0= & \int_{t_{0}}^{b}\left[f^{\top} R_{0} w+\sum_{k=1}^{L}(-1)^{k}\left(f^{\top} R_{k}\right)^{(k)} w\right] d t \\
& +\left[\sum_{k=1}^{L} \sum_{j=0}^{k-1}(-1)^{j}\left(f^{\top} R_{k}\right)^{(j)} w^{(k-1-j)}\right]_{t_{0}}^{b} .
\end{aligned}
$$

We now show that the first term in this expression equals $\int_{a}^{b} f^{\top} R\left(\frac{d}{d t}\right)^{*} w d t$. From the closed form expression for $R\left(\frac{d}{d t}\right)^{*}$ it follows that

$$
\begin{aligned}
f^{\top} R\left(\frac{d}{d t}\right)^{*}= & \sum_{k=0}^{L}(-1)^{k} \sum_{i=0}^{k}\left(\begin{array}{c}
k \\
i
\end{array}\right)\left(\frac{d^{k-i}}{d t^{k-i}} f\right)^{\top} R_{k}^{(i) \top} \\
& =f^{\top} R_{0}+\sum_{k=1}^{L}(-1)^{k} \sum_{i=0}^{k}\left(\begin{array}{c}
k \\
i
\end{array}\right)\left(\frac{d^{k-i}}{d t^{k-i}} f\right)^{\top} R_{k}^{(i) \top} .
\end{aligned}
$$

The formula for the higher derivative of a product reads

$$
\left(f^{\top} R_{k}\right)^{(k)}=\sum_{i=0}^{k}\left(\begin{array}{c}
k \\
i
\end{array}\right)\left(\frac{d^{k-i}}{d t^{k-i}} f\right)^{\top} R_{k}^{(i) \top},
$$

and consequently $f^{\top} R\left(\frac{d}{d t}\right)^{*}=f^{\top} R_{0}+\sum_{k=1}^{L}(-1)^{k}\left(f^{\top} R_{k}\right)^{(k)}$, which yields the desired equality. It follows that the first term in (3.7) equals 0 . It follows that $(0 \wedge w)(\cdot) \in \mathcal{B}$ if and only if for every $f(\cdot) \in \mathcal{F}_{t_{0}}\left(\mathbb{I}\left(t_{0}\right)\right)$ it holds that

$$
\left[\sum_{k=1}^{L} \sum_{j=0}^{k-1}(-1)^{j}\left(f^{\top} R_{k}\right)^{(j)} w^{(k-1-j)}\right]_{t_{0}}^{b}=0 .
$$

Use $\left(f^{\top} R_{k}\right)^{(j)}=\sum_{i=0}^{j}\left(\begin{array}{c}j \\ i\end{array}\right) f^{(j-i) \top} R_{k}^{(i)}, j=1, \ldots$, and the fact that $f(\cdot) \in \mathcal{F}_{0}\left(\mathbb{I}\left(t_{0}\right)\right)$ to conclude that $w(\cdot) \in \mathcal{B}$ is concatenable with zero at $t_{0}$ if and only if

$$
\left[\sum_{k=1}^{L} \sum_{j=0}^{k-1}(-1)^{j}\left(\sum_{i=0}^{j}\left(\begin{array}{l}
j \\
i
\end{array}\right) f^{(j-i) \top} R_{k}^{(i)}\right) w^{(k-1-j)}\right]_{t_{0}}^{b}
$$

$$
=-\sum_{k=1}^{L} \sum_{j=0}^{k-1}(-1)^{j}\left(\sum_{i=0}^{j}\left(\begin{array}{l}
j \\
i
\end{array}\right) f^{(j-i)}\left(t_{0}\right)^{\top} R_{k}^{(i)}\left(t_{0}\right)\right) w^{(k-1-j)\left(t_{0}\right)}=0 .
$$

We proceed to rewrite the expression

$$
\sum_{k=1}^{L} \sum_{j=0}^{k-1}(-1)^{j}\left(\sum_{i=0}^{j}\left(\begin{array}{l}
j \\
i
\end{array}\right) f^{(j-i) \top} R_{k}^{(i)}\right) w^{(k-1-j)}
$$

as a sum of terms involving $f(\cdot)^{(m)}, m=0, \ldots,(L-1)$-th, multiplying sums of terms involving the coefficients $R_{k}$ and $w(\cdot)^{(m)}, m=0, \ldots,(L-1)$. For $m:=j-i=0$, 
$f(\cdot)^{(m) \top}$ multiplies $\sum_{k=1}^{L} \sum_{j=0}^{k-1}(-1)^{j} R_{k}^{(j)} w(\cdot)^{(k-1-j)}$. For $m=j-i=1, f(\cdot)^{(m) \top}$ multiplies $\sum_{k=2}^{L} \sum_{j=1}^{k-1}\left({ }_{j-1}^{j}\right)(-1)^{j} R_{k}^{(j-1)} w(\cdot)^{(k-1-j)}$. An induction argument shows that the $m$-th derivative of $f(\cdot)$ multiplies

$$
\sum_{k=m+1}^{L} \sum_{j=m}^{k-1}\left(\begin{array}{c}
j \\
j-m
\end{array}\right)(-1)^{j} R_{k}^{(j-m)} w(\cdot)^{(k-1-j)} .
$$

Given the arbitrariness of $f(\cdot)$ in $\mathcal{F}_{0}\left(\mathbb{I}\left(t_{0}\right)\right)$, it follows that $0 \wedge_{t_{0}} w(\cdot) \in \mathcal{B}$ if and only if

$$
\sum_{k=m+1}^{L} \sum_{j=m}^{k-1}\left(\begin{array}{c}
j \\
j-m
\end{array}\right)(-1)^{j} R_{k}^{(j-m)}\left(t_{0}\right) w^{(k-1-j)}\left(t_{0}\right)=X_{m}\left(\frac{d}{d t}\right) w\left(t_{0}\right)=0,
$$

$m=0, \ldots, L-1$. This proves the claim.

REMARK 2. In the time-invariant case $R_{k}^{(j-m)}=0$ for $j-m \geq 1$. It follows from Th. 3.2 that $\left(0 \hat{t}_{0} w\right)(\cdot) \in \mathcal{B}$ if and only if $\sum_{k=m+1}^{L}(-1)^{j} R_{k} w^{(k-1-m)}\left(t_{0}\right)=0$, $m=0, \ldots, L-1$. This equivalence is Prop. 6.1 p. 1063 in [15].

In the following, if $P_{i}\left(\frac{d}{d t}\right), i=1, \ldots, N$, are polynomial differential operators with the same number of columns, we denote by $\operatorname{col}\left(P_{i}\left(\frac{d}{d t}\right)\right)_{i=1, \ldots, N}$ the polynomial differential operator obtained stacking the $P_{i}\left(\frac{d}{d t}\right)$ 's on top of each other.

Corollary 3.3. Let (2.1) be a kernel representation of $\mathcal{B}$. Define $X_{m}\left(\frac{d}{d t}\right)$ by (3.3). Let $w(\cdot) \in \mathcal{B}$, and define the trajectory of the auxiliary variable $x$ by

$$
x(\cdot):=\operatorname{col}\left(X_{m}\left(\frac{d}{d t}\right) w(\cdot)\right)_{m=0, \ldots, L-1},
$$

and the set of trajectories

$$
\mathcal{B}_{f}:=\{(w(\cdot), x(\cdot)) \mid w(\cdot) \in \mathcal{B} \text { and } x(\cdot) \text { is defined by }(3.8)\} .
$$

The variable $x$ is a state variable for $\mathcal{B}_{f}$, and $\mathcal{B}_{f}$ is a state system for $\mathcal{B}$.

Proof. Let $w_{i}(\cdot) \in \mathcal{B}, i=1,2$, and define the corresponding trajectories $x_{i}(\cdot)$, $i=1,2$ by (3.8). It follows from Th. 3.2 and Prop. 3.1 that the full trajectories $\left(w_{i}(\cdot), x_{i}(\cdot)\right), i=1,2$ have the following property: if $x_{1}(\cdot)$ and $x_{2}(\cdot)$ are continuous at $t_{0}$ and if $x_{1}\left(t_{0}\right)=x_{2}\left(t_{0}\right)$, then the concatenability conditions on $w_{1}(\cdot)$ and $w_{2}(\cdot)$ are satisfied. It follows that the external trajectory $w_{1}(\cdot) \hat{t}_{t_{0}} w_{2}(\cdot) \in \mathcal{B}$, and consequently that the concatenated trajectory $\left(w_{1}(\cdot), x_{1}(\cdot)\right) \underset{t_{0}}{\wedge}\left(w_{2}(\cdot), x_{2}(\cdot)\right)$ belongs to the set $\mathcal{B}_{f}$ defined by (3.9). Consequently, the variable $x$ defined by (3.8) satisfies the state property (Def. 2.1) and $\mathcal{B}_{f}$ is a state system for the external behavior $\mathcal{B}$.

REMARK 3. We discuss in order of appearance in the literature several approaches to the construction of state variables and state equations for linear time-varying systems.

In [24], state variables are introduced as auxiliary variables with respect to which first-order representations for a behavior can be computed (see Th. 8 p. $394 \mathrm{ibid}$.), and 
the state property is shown to be satisfied as a consequence of this (see sect. V.A p. 396). In formula (9) p. 395 of [24], it is shown that a state variable $x$ can be obtained from that of external variables $w$ applying to the latter a polynomial differential operator which coincides with that constructed from the differential operators (3.3). See also Rem. 5 below for a discussion on further parallels between the approach of [24] and the one presented in this paper.

The authors of [4] consider representations of the behavior where the entries of the coefficient matrices $R_{k}$ are smooth functions. They also view state variables as instrumental to achieving first-order representations, rather than starting from a first principles perspective. The state variable defined in [4] is precisely that induced by the polynomial differential operator (3.3), see formula (14) p. 723 ibid. The procedure yields a minimal state variable for the single-input, single-output case.

The approach to computation of state variables illustrated in [11] also proceeds from the realization problem rather than an intrinsic definition of state. The authors use the concept of left division in the non-commutative polynomial ring $\mathcal{M}[D]$ of polynomial differential operators with meromorphic coefficients, to arrive at formulas (10) p. 1953 and (13) p. 1954 to the same polynomial differential operator as (3.3). From the computational point of view, this approach is close to that of [15], in that the polynomial differential operators inducing a state variable are shown to be obtainable by repeated division of the polynomial matrices describing the system by the indeterminate corresponding to differentiation (see the definition of the "shift-and-cut" map in sect. 5, pp. 1060-ff. of [15]). The advantage of the approach of [11] over the aforementioned ones, including that illustrated in this paper, is that highlighting the connection of polynomial division and state construction brings computer algebra techniques to bear on the realisation problem.

4. State from latent variables: image representations. If two full trajectories $\left(w_{i}(\cdot), \ell_{i}(\cdot)\right), i=1,2$ satisfying (2.6) are concatenable at $t_{0}$, then also $w_{1}(\cdot) \hat{t}_{0} w_{2}(\cdot) \in \mathcal{B}$, the external behavior of (2.6). Since concatenability of full trajectories implies concatenability of the corresponding external ones, it follows that a state variable for $\mathcal{B}_{f}$ is also a state variable for the external behavior $\mathcal{B}$.

Now consider (2.6) as a kernel representation $\left[\begin{array}{ll}I_{q} & -M\left(\frac{d}{d t}\right)\end{array}\right]\left[\begin{array}{c}w(\cdot) \\ \ell(\cdot)\end{array}\right]=0$ of the full behavior $\mathcal{B}_{f}$. We now show that a state variable for $\mathcal{B}_{f}$ computed as in sect. 3 is a function of $\ell$ only.

COROLlary 4.1. If $\mathcal{B}$ is represented in image form (2.6) and $(w(\cdot), \ell(\cdot)) \in \mathcal{B}_{f}$, then the auxiliary variable $x$ with trajectories defined by

$$
\begin{aligned}
x(\cdot) & :=X\left(\frac{d}{d t}\right) \ell(\cdot) \\
& :=\operatorname{col}\left(-\sum_{k=i+1}^{N} \sum_{j=i}^{k-1}(-1)^{j}\left(\begin{array}{c}
j \\
j-i
\end{array}\right) M_{k}^{(j-i)} \ell^{(k-1-j)}(\cdot)\right)_{i=0, \ldots, N-1},
\end{aligned}
$$

is a state variable for $\mathcal{B}$, and the set of trajectories

$$
\mathcal{B}_{x}:=\left\{(w(\cdot), x(\cdot)) \mid(w(\cdot), \ell(\cdot)) \in \mathcal{B}_{f} \text { and } x(\cdot) \text { is defined by }(4.1)\right\},
$$

is a state system for $\mathcal{B}$. 
Proof. Let $(w(\cdot), \ell(\cdot)) \in \mathcal{B}_{f}$. Apply Th. 3.2 and Cor. 3.3 to conclude that the variable with trajectory defined by

$$
\begin{aligned}
X\left(\frac{d}{d t}\right)\left[\begin{array}{c}
w(\cdot) \\
\ell(\cdot)
\end{array}\right] & =\operatorname{col}\left(\sum_{k=i+1}^{N} \sum_{j=i}^{k-1}(-1)^{j}\left(\begin{array}{c}
j \\
j-i
\end{array}\right)\left[\begin{array}{ll}
0_{q \times q} & -M_{k}
\end{array}\right]^{(j-i)}\left[\begin{array}{c}
w^{(k-1-j)}(\cdot) \\
\ell^{(k-1-j)}(\cdot)
\end{array}\right]\right)_{i=0, \ldots, N-1} \\
& =\operatorname{col}\left(-\sum_{k=i+1}^{N} \sum_{j=i}^{k-1}(-1)^{j}\left(\begin{array}{c}
j \\
j-i
\end{array}\right) M_{k}^{(j-i)} \ell^{(k-1-j)}(\cdot)\right)_{i=0, \ldots, N-1},
\end{aligned}
$$

is a state variable for $\mathcal{B}_{f}$. The argument stated at the beginning of the section shows that a state variable for $\mathcal{B}_{f}$ is also a state variable for $\mathcal{B}$; the claim follows.

REMARK 4. In sect. 7 of [15] we proved that for (observable) representations (4.1) of a linear time-invariant differential system, concatenability of a full and an external trajectory are equivalent; consequently, the time-invariant equivalent of (4.1) provides a characterization of polynomial differential operators inducing a state variable. The result of Cor. 4.1 only provides a sufficient condition, but it is strong enough to allow us to study the relation between storage functions and state in sect. 6 of this paper. The converse implication will be considered elsewhere.

5. State and first order representations. In this section we prove that given a kernel representation of a behavior, it is possible to write down equations of first order in the state variable computed in (3.8) and zeroth order in the external variable.

TheOrem 5.1. Let $\mathcal{B}$ be a behavior described in kernel form by (2.1), and let $w(\cdot) \in \mathcal{B}$. Denote by $x$ the state variable defined by (3.8), with corresponding trajectory $x(\cdot)$. There exist $F, G \in \mathcal{M}^{p L \times p L}$ such that

$$
\frac{d}{d t} x(\cdot)=F x(\cdot)+G w(\cdot) .
$$

Proof. Denote by $x_{m}$ the $m$-th component of $x$ defined by (3.8), $m=0, \ldots, L-1$. In order to prove the claim, we prove the two equalities

$$
\begin{aligned}
\frac{d}{d t} x_{0}(\cdot)= & \sum_{k=1}^{L}(-1)^{k-1} R_{k}^{(k)} w(\cdot)-R_{0} w(\cdot) \\
\frac{d}{d t} x_{m}(\cdot)= & -x_{m-1}(\cdot)+(-1)^{m-1} R_{m} w(\cdot) \\
& +\sum_{k=m+1}^{L}(-1)^{k-1}\left(\begin{array}{c}
k \\
k-m
\end{array}\right) R_{k}^{(k-m)} w(\cdot) .
\end{aligned}
$$

$F$ and $G$ in (5.1) can be computed in a straightforward way from these identities.

To prove the first equality, since $x_{0}(\cdot)=\sum_{k=1}^{L} \sum_{j=0}^{k-1}(-1)^{j}\left(\begin{array}{c}j \\ j-0\end{array}\right) R_{k}^{(j)} w^{(k-1-j)}(\cdot)$ it follows that $\frac{d}{d t} x_{0}(\cdot)=\sum_{k=1}^{L} \sum_{j=0}^{k-1}(-1)^{j}\left[R_{k}^{(j+1)} w^{(k-1-j)}(\cdot)+R_{k}^{(j)} w^{(k-j)}(\cdot)\right]$. Define $j^{\prime}:=j+1$; then

$$
\sum_{k=1}^{L} \sum_{j=0}^{k-1}(-1)^{j} R_{k}^{(j+1)} w(\cdot)^{(k-1-j)}=\sum_{k=1}^{L} \sum_{j^{\prime}=1}^{k}(-1)^{j^{\prime}-1} R_{k}^{\left(j^{\prime}\right)} w(\cdot)^{\left(k-j^{\prime}\right)},
$$


$311 \sum_{k=1}^{L} \sum_{j^{\prime}=1}^{k}(-1)^{j^{\prime}-1} R_{k}^{\left(j^{\prime}\right)} w(\cdot)^{\left(k-j^{\prime}\right)}=\sum_{k=1}^{L}(-1)^{k-1} R_{k}^{(k)} w(\cdot)+\sum_{k=2}^{L} \sum_{j^{\prime}=1}^{k-1}(-1)^{j^{\prime}-1} R_{k}^{\left(j^{\prime}\right)} w(\cdot)^{\left(k-j^{\prime}\right)}$.

$$
\frac{d}{d t} x_{0}(\cdot)=\sum_{k=1}^{L} \sum_{j^{\prime}=1}^{k}(-1)^{j^{\prime}-1} R_{k}^{\left(j^{\prime}\right)} w(\cdot)^{\left(k-j^{\prime}\right)}+\sum_{k=1}^{L} \sum_{j=0}^{k-1}(-1)^{j} R_{k}^{(j)} w(\cdot)^{(k-j)} .
$$

Separating the term with $j^{\prime}=k$ in the inner sum of the first term in (5.3) we obtain

and consequently

The second expression in (5.3) can be rewritten as

$$
\sum_{k=1}^{L} \sum_{j=0}^{k-1}(-1)^{j} R_{k}^{(j)} w(\cdot)^{(k-j)}=\sum_{k=1}^{L} R_{k} w(\cdot)^{(k)}+\sum_{k=2}^{L} \sum_{j=1}^{k-1}(-1)^{j} R_{k}^{(j)} w(\cdot)^{(k-j)}
$$

Consequently, $\frac{d}{d t} x_{0}(\cdot)=\sum_{k=1}^{L}(-1)^{k-1} R_{k}^{(k)} w(\cdot)+\sum_{k=1}^{L} R_{k} w^{(k)}(\cdot)$ equals

$$
\sum_{k=1}^{L}(-1)^{k-1} R_{k}^{(k)} w(\cdot)+\underbrace{\sum_{k=1}^{L} R_{k} w^{(k)}(\cdot)+R_{0} w(\cdot)}_{=R\left(\frac{d}{d t}\right) w(\cdot)=0}-R_{0} w=\sum_{k=1}^{L}(-1)^{k-1} R_{k}^{(k)} w(\cdot)-R_{0} w(\cdot)
$$

The first equality in (5.2) is proved. As for the second equality in (5.2), observe that

$$
\begin{aligned}
\frac{d}{d t} x_{m}(\cdot)= & \sum_{k=m+1}^{L} \sum_{j=m}^{k-1}(-1)^{j}\left(\begin{array}{c}
j \\
j-m
\end{array}\right) R_{k}^{(j-m+1)} w(\cdot)^{(k-1-j)} \\
& +\sum_{k=m+1}^{L} \sum_{j=m}^{k-1}(-1)^{j}\left(\begin{array}{c}
j \\
j-m
\end{array}\right) R_{k}^{(j-m)} w(\cdot)^{(k-j)} .
\end{aligned}
$$

Define $j^{\prime}:=j+1$ and rewrite the first expression on the right-hand side as

(1)

$$
\begin{aligned}
& \sum_{k=m+1}^{L} \sum_{j=m}^{k-1}(-1)^{j}\left(\begin{array}{c}
j \\
j-m
\end{array}\right) R_{k}^{(j-m+1)} w(\cdot)^{(k-1-j)} \\
= & \sum_{k=m+1}^{L} \sum_{j^{\prime}=m+1}^{k}(-1)^{j^{\prime}-1}\left(\begin{array}{c}
j^{\prime}-1 \\
j^{\prime}-1-m
\end{array}\right) R_{k}^{\left(j^{\prime}-m\right)} w(\cdot)^{\left(k-j^{\prime}\right)} .
\end{aligned}
$$

Recall Pascal's identity $\left(\begin{array}{c}j^{\prime}-1 \\ j^{\prime}-m-1\end{array}\right)=\left(\begin{array}{c}j^{\prime} \\ j^{\prime}-m\end{array}\right)-\left(\begin{array}{c}j^{\prime}-1 \\ j^{\prime}-m\end{array}\right)$ and conclude that

$$
\begin{aligned}
\frac{d}{d t} x_{m}(\cdot)= & \sum_{k=m+1}^{L} \sum_{j^{\prime}=m+1}^{k}(-1)^{j^{\prime}-1}\left[\left(\begin{array}{c}
j^{\prime} \\
j^{\prime}-m
\end{array}\right)-\left(\begin{array}{c}
j^{\prime}-1 \\
j^{\prime}-m
\end{array}\right)\right] R_{k}^{\left(j^{\prime}-m\right)} w(\cdot)^{\left(k-j^{\prime}\right)} \\
& +\sum_{k=m+1}^{L} \sum_{j=m}^{k-1}(-1)^{j}\left(\begin{array}{c}
j \\
j-m
\end{array}\right) R_{k}^{(j-m)} w(\cdot)^{(k-j)}
\end{aligned}
$$


To arrive at the second equality in (5.2), consider first that for $k$ between $m+1$ and $L$ it holds that

$$
\begin{aligned}
& \sum_{j^{\prime}=m+1}^{k}(-1)^{j^{\prime}}\left(\begin{array}{c}
j^{\prime} \\
j^{\prime}-m
\end{array}\right) R_{k}^{\left(j^{\prime}-m\right)} w(\cdot)^{\left(k-j^{\prime}\right)}+\sum_{j=m}^{k-1}(-1)^{j}\left(\begin{array}{c}
j \\
j-m
\end{array}\right) R_{k}^{(j-m)} w(\cdot)^{(k-j)} \\
= & (-1)^{k-1}\left(\begin{array}{c}
k \\
k-m
\end{array}\right) R_{k}^{(k-m)} w(\cdot)+(-1)^{m}\left(\begin{array}{c}
m \\
0
\end{array}\right) R_{k} w(\cdot)^{(k-m)} .
\end{aligned}
$$

Conclude from this equality that

$$
\begin{aligned}
\frac{d}{d t} x_{m}(\cdot)= & \sum_{k=m+1}^{L}\left[(-1)^{k-1}\left(\begin{array}{c}
k \\
k-m
\end{array}\right) R_{k}^{(k-m)} w(\cdot)+(-1)^{m}\left(\begin{array}{c}
m \\
0
\end{array}\right) R_{k} w(\cdot)^{(k-m)}\right] \\
& -\sum_{k=m+1}^{L} \sum_{j^{\prime}=m+1}^{k}(-1)^{j^{\prime}-1}\left(\begin{array}{c}
j^{\prime}-1 \\
j^{\prime}-m
\end{array}\right) R_{k}^{\left(j^{\prime}-m\right)} w(\cdot)^{\left(k-j^{\prime}\right)} .
\end{aligned}
$$

We now prove that this expression equals $-x_{m-1}(\cdot)$ + zeroth-order terms in $w(\cdot)$.

To do this, defining $j^{\prime}:=j+1$, we rewrite the expression (3.3) for $x_{m-1}(\cdot)$ as

$$
\begin{aligned}
x_{m-1}(\cdot)= & \sum_{k=m}^{L} \sum_{j^{\prime}=m}^{k}(-1)^{j^{\prime}-1}\left(\begin{array}{c}
j^{\prime}-1 \\
j^{\prime}-m
\end{array}\right) R_{k}^{\left(j^{\prime}-m\right)} w(\cdot)^{\left(k-j^{\prime}\right)} \\
= & (-1)^{m-1}\left(\begin{array}{c}
m-1 \\
0
\end{array}\right) R_{m} w(\cdot)+\sum_{k=m+1}^{L} \sum_{j^{\prime}=m}^{k}(-1)^{j^{\prime}-1}\left(\begin{array}{c}
j^{\prime}-1 \\
j^{\prime}-m
\end{array}\right) R_{k}^{\left(j^{\prime}-m\right)} w(\cdot)^{\left(k-j^{\prime}\right)} \\
= & (-1)^{m-1}\left(\begin{array}{c}
m-1 \\
0
\end{array}\right) R_{m} w(\cdot)+\sum_{k=m+1}^{L}(-1)^{m-1}\left(\begin{array}{c}
m-1 \\
0
\end{array}\right) R_{k} w(\cdot)^{(k-m)} \\
& +\sum_{k=m+1}^{L} \sum_{j^{\prime}=m+1}^{k}(-1)^{j^{\prime}-1}\left(\begin{array}{c}
j^{\prime}-1 \\
j^{\prime}-m
\end{array}\right) R_{k}^{\left(j^{\prime}-m\right)} w(\cdot)^{\left(k-j^{\prime}\right)} .
\end{aligned}
$$

The second and third term of this expression are the opposite of the second and third term in (5.5). Conclude that

$$
\begin{aligned}
\frac{d}{d t} x_{m}(\cdot)= & -x_{m-1}(\cdot)+(-1)^{m-1}\left(\begin{array}{c}
m-1 \\
0
\end{array}\right) R_{m} w(\cdot) \\
& +\sum_{k=m+1}^{L}(-1)^{(k-1)}\left(\begin{array}{c}
k \\
k-m
\end{array}\right) R_{k}^{(k-m)} w(\cdot) .
\end{aligned}
$$

The claim of the theorem is proved.

In the following remarks we discuss alternative methods for the computation of first order equations, and some further work opened up by the result of Th. 5.1.

REMARK 5. In Th. 8 p. 394 of [24], a procedure is given to compute a special ("output nulling") first-order-in- $x$, zeroth-order-in- $w$ representation for a behavior in kernel form:

$$
\begin{aligned}
\frac{d}{d t} x(\cdot) & =F x(\cdot)+G w(\cdot) \\
0 & =M x(\cdot)+N w(\cdot),
\end{aligned}
$$


where $F, M$ are real matrices with $(F, M)$ an observable pair, and $G, N$ are matrices with meromorphic entries. In sect. $\mathrm{V}$ ibid. it is shown how to compute from (5.6) an input-output-state representation associated with a time-varying quadruple $(A, B, C, D)$ with $(A, C)$ observable.

In [4] it is shown that in the single-input, single-output case the state variable (3.8) can be used to compute an observability canonical form representation (with matrix entries being ratios of smooth functions) of the behavior, see formulas (37)-(38) p. 728 therein.

The authors of [11] consider the multiple-input, multiple-output case, and obtain explicit formulas to compute a matrix quadruple $(A, B, C, D)$ with meromorphic entries describing the system in observability canonical form, see Th. 3.1 p. 1953.

REMARK 6. We showed how representations of first order in the state and zeroth order in the external variables can be computed in our approach. However, the result of Th. 5.1 falls short of being completely satisfactory on various accounts; we now summarise the most pressing directions for further research. The first one is how to compute input-state-output representations in our approach (see also Rem. 5). The second one is to characterize all state variables for kernel representations on the basis of Def. 2.1 and Cor. 3.3. This development would open up further interesting research questions, among them minimality and the computation of canonical representations (e.g. observability, controllability). Further extensions are the computation of state variables and representations starting from hybrid (but not image) representations (2.3).

6. State and storage functions. We analyse the relation of the notion of state proposed in this paper with the notion of storage function introduced in the framework for dissipativity of [18] and further studied in [5, 20]. We consider quadratic functionals defined on the external trajectories of a system, induced by $S=S^{\top} \in$ $\mathbb{R}^{q \times q}$, and defined by

$$
w(\cdot) \rightarrow w(\cdot)^{\top} S w(\cdot)=: Q_{S}(w(\cdot)) .
$$

Our analysis of dissipative systems is local, based as it is on the interplay of solutions and quadratic functionals on finite intervals $\left[t_{0}, t_{1}\right] \subset \mathbb{R}$. In this way we circumvent the integrability difficulties inherent in considering dissipative systems over the halfor full time set $\mathbb{R}$. To make progress on the general case, it makes sense to consider the simpler local one; see [5] for a different approach in an operator-theoretic setting.

In the following we consider systems in image form (2.6). In this case, one can rewrite the quadratic functional $w(\cdot) \rightarrow w(\cdot)^{\top} S w(\cdot)=: Q_{S}(w(\cdot))$ as a quadratic functional acting on $\ell(\cdot)$ and its derivatives:

$$
\begin{aligned}
w(\cdot)^{\top} S w(\cdot) & =\left(M\left(\frac{d}{d t}\right) \ell(\cdot)\right)^{\top} S\left(M\left(\frac{d}{d t}\right) \ell(\cdot)\right) \\
& =\sum_{i, j=0}^{L}\left(M_{i} \frac{d^{i} \ell(\cdot)}{d t^{i}}\right)^{\top} S\left(M_{j} \frac{d^{j} \ell(\cdot)}{d t^{j}}\right)=: Q_{\Phi}(\ell(\cdot)) .
\end{aligned}
$$

REMARK 7. By considering only systems representable in image form, we restrict our investigation to controllable systems (see the discussion following eq. (2.6)). In this we follow the approach of [20], where dissipativity for higher-order linear timeinvariant systems was introduced, and the relation between storage functions and state functions was first elucidated. 
However, the existence of passive, non-controllable electrical circuits (see $[1,6]$ ) shows that there is no intrinsic relation between dissipativity and controllability. In the context of systems described by higher-order constant-coefficient linear differential equations, in [6] the authors provided necessary and sufficient conditions on the uncontrollable part of the behavior for a given system to be passive as defined in Def. 5 ibid.. Our concern is with higher-order linear, time-varying cyclo-dissipative systems; for the definition of cyclo-dissipativity, see Def. 6.1 in sect. 6.1 of the present paper. How to characterise cyclo-dissipativity for the case of uncontrollable linear time-invariant systems is discussed in Remarks 8 and 9 on pp. 1722-1724 of [20], where an alternative definition is proposed.

In [14] the authors study uncontrollable cyclo-dissipative systems described by higher-order constant-coefficient linear differential equations, in accordance with the aforementioned definition in [20]. In Cor. 5.6 of [14] it is proved that, under an "unmixing" assumption on the poles of the uncontrollable part of the behavior, the storage function is a quadratic function of the state also in the uncontrollable case. The extension of such results to the time-varying case is an open problem.

6.1. Cyclo-dissipativity and cyclo-losslessness. The following definition is analogous to Def. 8 p. 334 of [5].

Definition 6.1. Let $\mathcal{B}=\operatorname{im} M\left(\frac{d}{d t}\right)$, with $M\left(\frac{d}{d t}\right) \in \mathcal{M}^{q \times m}\left[\frac{d}{d t}\right]$, as in (2.6). Denote by $X\left(\frac{d}{d t}\right)$ the polynomial differential operator defined in (4.1).

$\mathcal{B}$ is cyclo-dissipative with respect to $Q_{S}$ if for every $\left[t_{0}, t_{1}\right] \subset \mathbb{R}$ such that $\left[t_{0}, t_{1}\right] \subseteq$ $\cap_{k=0}^{L} \operatorname{dom}\left(M_{k}\right)$, and every $\ell \in \mathcal{C}^{\infty}\left(\left[t_{0}, t_{1}\right], \mathbb{R}^{m}\right)$ such that $X\left(\frac{d}{d t}\right) \ell\left(t_{i}\right)=0, i=0,1$, it holds that

$$
\int_{t_{0}}^{t_{1}} w(\tau)^{\top} S w(\tau) d \tau \geq 0
$$

If this inequality holds, then $Q_{S}$ is called a supply rate for $\mathcal{B}$.

Interpreting the supply rate $Q_{S}$ as input power, the inequality (6.2) states that a net absorption of energy occurs along every system trajectory beginning and ending "at rest", expressed by the conditions $X\left(\frac{d}{d t}\right) \ell\left(t_{i}\right)=0, i=0,1$ on the state of the system at the extremes of integration.

The following definition is analogous to Def. 13 p. 345 of [5].

Definition 6.2. Let $\mathcal{B}=\operatorname{im} M\left(\frac{d}{d t}\right)$, with $M\left(\frac{d}{d t}\right) \in \mathcal{M}^{q \times m}\left[\frac{d}{d t}\right]$, as in (2.6). Denote by $X\left(\frac{d}{d t}\right)$ the polynomial differential operator defined in (4.1).

$\mathcal{B}$ is called cyclo-lossless with respect to the supply rate $Q_{S}$ if for every $\left[t_{0}, t_{1}\right] \subset \mathbb{R}$ such that $\left[t_{0}, t_{1}\right] \subseteq \cap_{k=0}^{L} \operatorname{dom}\left(M_{k}\right)$, and for every $\ell(\cdot) \in \mathcal{C}^{\infty}\left(\left[t_{0}, t_{1}\right], \mathbb{R}^{m}\right)$ such that $X\left(\frac{d}{d t}\right) \ell\left(t_{i}\right)=0, i=0,1$, it holds that

$$
\int_{t_{0}}^{t_{1}} w(\tau)^{\top} S w(\tau) d \tau=0
$$

It follows from Def.s 6.1 and 6.2 that a cyclo-lossless system is also cyclo-dissipative. If we interpret the supply rate $Q_{S}$ as input power, then cyclo-losslessness is equivalent to path independence of the integral of $Q_{S}$.

Eq. (6.1) shows that when dealing with systems described in image form and supply rates induced by constant matrices, it is natural to study quadratic functionals of the latent variable $\ell$ and its higher-order derivatives. The next section introduces some important concepts in this framework. 
6.2. Bilinear and quadratic differential forms. We introduce the notion of bilinear-and quadratic differential form with time-varying coefficients (see [20] for the time-invariant case).

Let $\Phi_{i, j} \in \mathcal{M}^{n_{1} \times n_{2}}, i, j=0, \ldots, L$ be a family of meromorphic matrix functions. Let $\left[t_{0}, t_{1}\right] \subseteq \bigcap_{i, j=0}^{L} \operatorname{dom}\left(\Phi_{i, j}\right)$, and associate with $\left\{\Phi_{i, j}\right\}_{i, j=0, \ldots, L}$ the form

$$
\begin{aligned}
B_{\Phi}: \mathcal{C}^{\infty}\left(\left[t_{0}, t_{1}\right], \mathbb{R}^{n_{1}}\right) \times \mathcal{C}^{\infty}\left(\left[t_{0}, t_{1}\right], \mathbb{R}^{n_{2}}\right) & \rightarrow \mathcal{C}^{\infty}\left(\left[t_{0}, t_{1}\right], \mathbb{R}\right) \\
\left(\ell_{1}(\cdot), \ell_{2}(\cdot)\right) & \rightarrow \sum_{i, j=0}^{L}\left(\frac{d^{i}}{d t^{i}} \ell_{1}(\cdot)\right)^{\top} \Phi_{i, j}\left(\frac{d^{j}}{d t^{j}} \ell_{2}(\cdot)\right) .
\end{aligned}
$$

It is straightforward to see that $B_{\Phi}$ is bilinear. If $n_{1}=n_{2}=: m$, then we also associate to $\left\{\Phi_{i, j}\right\}_{i, j=0, \ldots, L}$ the quadratic form

$$
\begin{aligned}
Q_{\Phi}: \mathcal{C}^{\infty}\left(\left[t_{0}, t_{1}\right], \mathbb{R}^{m}\right) & \rightarrow \mathcal{C}^{\infty}\left(\left[t_{0}, t_{1}\right], \mathbb{R}\right) \\
\ell(\cdot) & \rightarrow \sum_{i, j=0}^{L}\left(\frac{d^{i}}{d t^{i}} \ell(\cdot)\right)^{\top} \Phi_{i, j}\left(\frac{d^{j}}{d t^{j}} \ell(\cdot)\right) .
\end{aligned}
$$

In the following when considering bilinear and quadratic differential forms we assume that $\Phi_{i, j}=\Phi_{j, i}^{\top}, i, j=0, \ldots, L$.

We associate to the bilinear differential form $B_{\Phi}$ in (6.4) its infinite coefficient matrix (with only a finite number of nonzero entries!)

$$
\widetilde{\Phi}:=\left[\begin{array}{cccccc}
\Phi_{0,0} & \Phi_{0,1} & \ldots & \Phi_{0, L} & 0_{m \times m} & \ldots \\
\Phi_{1,0} & \Phi_{1,1} & \ldots & \Phi_{1,2} & 0_{m \times m} & \ldots \\
\vdots & \vdots & \ldots & \vdots & \vdots & \ldots \\
\Phi_{L, 0} & \Phi_{L, 1} & \ldots & \Phi_{L, L} & 0_{m \times m} & \ldots \\
0_{m \times m} & 0_{m \times m} & \ldots & 0_{m \times m} & 0_{m \times m} & \ldots \\
\vdots & \vdots & \ldots & \vdots & \vdots & \ldots
\end{array}\right]
$$

in the sense that if $\ell(\cdot) \in \mathcal{C}^{\infty}\left(\left[t_{0}, t_{1}\right], \mathbb{R}^{m}\right)$ and we define $\operatorname{jet}(\ell(\cdot)):=\operatorname{col}\left(\frac{d^{i}}{d t^{i}} \ell(\cdot)\right)_{i=0, \ldots}$, then

$$
B_{\Phi}\left(\ell_{1}(\cdot), \ell_{2}(\cdot)\right)=\sum_{i, j=0}^{L}\left(\frac{d^{i} \ell_{1}(\cdot)}{d t^{i}}\right)^{\top} \Phi_{i, j}\left(\frac{d^{j} \ell_{2}(\cdot)}{d t^{j}}\right)=\operatorname{jet}\left(\ell_{1}(\cdot)\right)^{\top} \widetilde{\Phi} \operatorname{jet}\left(\ell_{2}(\cdot)\right) .
$$

It is straightforward to verify that the association between bilinear and quadratic differential forms and their coefficient matrices is bijective.

Define the entry-wise derivative of $M \in \mathcal{M}^{m \times m}$ by $\left(\frac{d}{d t} M\right)_{i, j}:=\frac{d}{d t}\left(M_{i, j}\right), i, j=$ $1, \ldots, m$. On the coefficient matrix (6.5) we define the entry-wise differentiation operation, defined by

$$
\frac{d}{d t} \widetilde{\Phi}:=\left[\begin{array}{cccccc}
\frac{d}{d t} \Phi_{0,0} & \frac{d}{d t} \Phi_{0,1} & \ldots & \frac{d}{d t} \Phi_{0, L} & 0_{m \times m} & \ldots \\
\frac{d}{d t} \Phi_{1,0} & \frac{d}{d t} \Phi_{1,1} & \ldots & \frac{d}{d t} \Phi_{1,2} & 0_{m \times m} & \ldots \\
\vdots & \vdots & \ldots & \vdots & \vdots & \ldots \\
\frac{d}{d t} \Phi_{L, 0} & \frac{d}{d t} \Phi_{L, 1} & \ldots & \frac{d}{d t} \Phi_{L, L} & 0_{m \times m} & \ldots \\
\vdots & \vdots & \ldots & \vdots & \vdots & \ldots
\end{array}\right] .
$$


We also define the down- and right-shift operations, respectively denoted by $\sigma_{D}$ and $\sigma_{R}$, respectively by$$
\sigma_{D}(\widetilde{\Phi}):=\left[\begin{array}{cccccc}
0_{m \times m} & 0_{m \times m} & \ldots & 0_{m \times m} & 0_{m \times m} & \ldots \\
\Phi_{0,0} & \Phi_{0,1} & \ldots & \Phi_{0, L} & 0_{m \times m} & \ldots \\
\Phi_{1,0} & \Phi_{1,1} & \ldots & \Phi_{1,2} & 0_{m \times m} & \ldots \\
\vdots & \vdots & \ldots & \vdots & \vdots & \ldots \\
\Phi_{L, 0} & \Phi_{L, 1} & \ldots & \Phi_{L, L} & 0_{m \times m} & \ldots \\
\vdots & \vdots & \ldots & \vdots & \vdots & \ldots
\end{array}\right],
$$

and

$$
\sigma_{R}(\widetilde{\Phi}):=\left[\begin{array}{ccccccc}
0_{m \times m} & \Phi_{0,0} & \Psi_{0,1} & \ldots & \Phi_{0, L} & 0_{m \times m} & \ldots \\
0_{m \times m} & \Phi_{1,0} & \Psi_{1,1} & \ldots & \Phi_{1,2} & 0_{m \times m} & \ldots \\
\vdots & \vdots & \vdots & \ldots & \vdots & \vdots & \ldots \\
0_{m \times m} & \Phi_{L, 0} & \Phi_{L, 1} & \ldots & \Phi_{L, L} & 0_{m \times m} & \ldots \\
\vdots & \vdots & \ldots & \vdots & \vdots & \ldots &
\end{array}\right] .
$$

Define the derivative of the bilinear differential form $B_{\Phi}$, denoted by $\mathcal{B}_{\dot{\Phi}}$, by

$$
\frac{d}{d t} B_{\Phi}\left(\ell_{1}(\cdot), \ell_{2}(\cdot)\right):=\sum_{i, j=0}^{L} \frac{d}{d t}\left[\left(\frac{d^{i} \ell_{1}(\cdot)}{d t^{i}}\right)^{\top} \Phi_{i, j}\left(\frac{d^{j} \ell_{2}(\cdot)}{d t^{j}}\right)\right] ;
$$

it is straightforward to verify that $\frac{d}{d t} B_{\Phi}$ is also a bilinear differential form. Use Leibniz's rule for differentiation to verify that the coefficient matrix of $\mathcal{B}_{\dot{\Phi}}$ is

$$
\widetilde{\dot{\Phi}}=\frac{d}{d t} \widetilde{\Phi}+\sigma_{D}(\widetilde{\Phi})+\sigma_{R}(\widetilde{\Phi})
$$

6.3. Storage functions. We recall the notion of storage function.

Definition 6.3. Assume that the system (2.6) is cyclo-dissipative with respect to a supply rate $Q_{S}$. A quadratic differential form $Q_{\Psi}$ is a storage function if for every $w(\cdot) \in \mathcal{B}$ and every $\left[t_{0}, t_{1}\right] \subset \operatorname{dom}(w(\cdot))$ it holds that

$$
\int_{t_{0}}^{t_{1}} w(\tau)^{\top} S w(\tau) d \tau \geq Q_{\Psi}(w)\left(t_{1}\right)-Q_{\Psi}(w)\left(t_{0}\right) .
$$

In the rest of this section we first give several characterizations of cyclo-losslessness for systems in image form, and we prove the existence of a storage function for such systems. Moreover, we prove that such storage function is a quadratic function of the state of the system. Lastly, we show that the results for cyclo-lossless systems apply also to cyclo-dissipative systems. We begin with the following instrumental result.

Lemma 6.4. Let $M\left(\frac{d}{d t}\right)=M_{0}+M_{1} \frac{d}{d t}+\ldots+M_{L} \frac{d^{L}}{d t^{L}}$ be a polynomial differential operator with $q \times m$ meromorphic coefficients, and $S=S^{\top} \in \mathbb{R}^{q \times q}$. Define

$$
X\left(\frac{d}{d t}\right):=\operatorname{col}\left(\sum_{k=i+1}^{N} \sum_{j=i}^{k-1}(-1)^{j}\left(\begin{array}{c}
j \\
j-i
\end{array}\right) M_{k}^{(j-i)} \frac{d^{k-1-j}}{d t^{k-1-j}}\right)_{i=0, \ldots, L-1} .
$$


For every $\left[t_{0}, t_{1}\right] \subset \mathbb{R},\left[t_{0}, t_{1}\right] \subseteq \cap_{k=0}^{L} \operatorname{dom}\left(M_{k}\right)$, and every $\ell_{i}(\cdot) \in \mathcal{C}^{\infty}\left(\left[t_{0}, t_{1}\right], \mathbb{R}^{m}\right)$, $i=1,2$, it holds that

$$
\begin{aligned}
& \int_{t_{0}}^{t_{1}}\left(M\left(\frac{d}{d t}\right) \ell_{1}(\cdot)\right)^{\top} S\left(M\left(\frac{d}{d t}\right) \ell_{2}(\cdot)\right) d t \\
& =\left.\left(X\left(\frac{d}{d t}\right) \ell_{1}(\cdot)\right)^{\top} \operatorname{col}\left(S \frac{d^{i}}{d t^{i}} M\left(\frac{d}{d t}\right) \ell_{2}(\cdot)\right)_{i=0, \ldots, L-1}\right|_{t_{0}} ^{t_{1}} \\
& +\int_{t_{0}}^{t_{1}} \ell_{1}(\cdot)^{\top}\left(M\left(\frac{d}{d t}\right)^{*} S M\left(\frac{d}{d t}\right) \ell_{2}(\cdot)\right) d t .
\end{aligned}
$$

Proof. From the definition of $M\left(\frac{d}{d t}\right)$ it follows that

$$
\left(M\left(\frac{d}{d t}\right) \ell_{1}(\cdot)\right)^{\top} S\left(M\left(\frac{d}{d t}\right) \ell_{2}(\cdot)\right)=\sum_{k=0}^{L} \ell_{1}(\cdot)^{(k) \top} M_{k}^{\top} S\left(M\left(\frac{d}{d t}\right) \ell_{2}(\cdot)\right) \text {; }
$$

now use integration by parts, as done in the proof of Th. 3.2, to conclude that

$$
\begin{aligned}
& \int_{t_{0}}^{t_{1}}\left(M\left(\frac{d}{d t}\right) \ell_{1}(\cdot)\right)^{\top} S\left(M\left(\frac{d}{d t}\right) \ell_{2}(\cdot)\right) d t \\
& =\int_{t_{0}}^{t_{1}} \ell_{1}(\cdot)^{\top} M_{0}^{\top} S\left(M\left(\frac{d}{d t}\right) \ell_{2}(\cdot)\right) d t \\
& +\int_{t_{0}}^{t_{1}} \sum_{k=1}^{L} \frac{d}{d t}\left[\sum_{j=0}^{k-1}(-1)^{j} \ell_{1}(\cdot)^{(k-1-j) \top}\left(M_{k}^{\top} S M\left(\frac{d}{d t}\right) \ell_{2}(\cdot)\right)^{(j)}\right] d t \\
& +\int_{t_{0}}^{t_{1}} \sum_{k=1}^{L}(-1)^{k} \ell_{1}(\cdot)^{\top}\left(M_{k}^{\top} S M\left(\frac{d}{d t}\right) \ell_{2}(\cdot)\right)^{(k)} d t .
\end{aligned}
$$

We now show that the sum of the first and the last terms on the right-hand side of the previous expression, i.e. $\int_{t_{0}}^{t_{1}} \sum_{k=0}^{L}(-1)^{k} \ell_{1}(\cdot)^{\top}\left(M_{k}^{\top} S M\left(\frac{d}{d t}\right) \ell_{2}(\cdot)\right)^{(k)} d t$, equals

$$
\int_{t_{0}}^{t_{1}} \ell_{1}(\cdot)^{\top}\left(M\left(\frac{d}{d t}\right)^{*} S M\left(\frac{d}{d t}\right) \ell_{2}(\cdot)\right) d t
$$

Apply Leibniz's rule for differentiation to conclude that

$$
\left(M_{k}^{\top} S M\left(\frac{d}{d t}\right) \ell_{2}(\cdot)\right)^{(k)}=\sum_{i=0}^{k}\left(\begin{array}{c}
k \\
i
\end{array}\right) M_{k}^{(k-i) \top} S \frac{d^{i}}{d t^{i}}\left(M\left(\frac{d}{d t}\right) \ell_{2}(\cdot)\right) .
$$

It follows that

$$
\begin{aligned}
& \int_{t_{0}}^{t_{1}} \sum_{k=0}^{L}(-1)^{k} \ell_{1}(\cdot)^{\top}\left(M_{k}^{\top} S M\left(\frac{d}{d t}\right) \ell_{2}(\cdot)\right)^{(k)} d t \\
& =\int_{t_{0}}^{t_{1}} \sum_{k=0}^{L}(-1)^{k} \ell_{1}(\cdot)^{\top} \sum_{i=0}^{k}\left(\begin{array}{c}
k \\
i
\end{array}\right) M_{k}^{(k-i) \top} S \frac{d^{i}}{d t^{i}}\left(M\left(\frac{d}{d t}\right) \ell_{2}(\cdot)\right) d t .
\end{aligned}
$$

Define $m:=k-i$, and rewrite the last expression as

$$
\int_{t_{0}}^{t_{1}} \sum_{k=0}^{L}(-1)^{k} \ell_{1}(\cdot)^{\top} \sum_{m=0}^{k}\left(\begin{array}{c}
k \\
k-m
\end{array}\right) M_{k}^{(m) \top} S \frac{d^{k-m}}{d t^{k-m}}\left(M\left(\frac{d}{d t}\right) \ell_{2}(\cdot)\right) d t .
$$


Recall that $M\left(\frac{d}{d t}\right)^{*}=\sum_{k=0}^{L}(-1)^{k} \sum_{m=0}^{k}\left(\begin{array}{c}k \\ m\end{array}\right) M_{k}^{(m)} \frac{d^{k-m}}{d t^{k-m}}$ and apply the binomial coefficient identity $\left(\begin{array}{c}k \\ k-m\end{array}\right)=\left(\begin{array}{c}k \\ m\end{array}\right)$ to conclude that

$$
\begin{aligned}
& \int_{t_{0}}^{t_{1}} \sum_{k=0}^{L}(-1)^{k} \ell_{1}(\cdot)^{\top}\left(M_{k}^{\top} S M\left(\frac{d}{d t}\right) \ell_{2}(\cdot)\right)^{(k)} d t \\
& =\int_{t_{0}}^{t_{1}} \ell_{1}(\cdot)^{\top}\left(M\left(\frac{d}{d t}\right)^{*} S M\left(\frac{d}{d t}\right) \ell_{2}(\cdot)\right) d t,
\end{aligned}
$$

as claimed.

From the equality just proved it follows that

$$
\begin{aligned}
& \int_{t_{0}}^{t_{1}}\left(M\left(\frac{d}{d t}\right) \ell_{1}(\cdot)\right)^{\top} S\left(M\left(\frac{d}{d t}\right) \ell_{2}(\cdot)\right) d t \\
& =\int_{t_{0}}^{t_{1}} \ell_{1}(\cdot)^{\top}\left(M\left(\frac{d}{d t}\right)^{*} S M\left(\frac{d}{d t}\right) \ell_{2}(\cdot)\right) d t \\
& +\int_{t_{0}}^{t_{1}} \sum_{k=1}^{L} \frac{d}{d t}\left[\sum_{j=0}^{k-1}(-1)^{j} \ell_{1}(\cdot)^{(k-1-j) \top}\left(M_{k}^{\top} S M\left(\frac{d}{d t}\right) \ell_{2}(\cdot)\right)^{(j)}\right] d t .
\end{aligned}
$$

To prove the claim of the Lemma it remains to prove that

$$
\begin{aligned}
& \int_{t_{0}}^{t_{1}} \sum_{k=1}^{L} \frac{d}{d t}\left[\sum_{j=0}^{k-1}(-1)^{j} \ell_{1}(\cdot)^{(k-1-j) \top}\left(M_{k}^{\top} S M\left(\frac{d}{d t}\right) \ell_{2}(\cdot)\right)^{(j)}\right] d t \\
& =\left.\sum_{k=1}^{L} \sum_{j=0}^{k-1}(-1)^{j} \ell_{1}(\cdot)^{(k-1-j) \top}\left(M_{k}^{\top} S M\left(\frac{d}{d t}\right) \ell_{2}(\cdot)\right)^{(j)}\right|_{t_{0}} ^{t_{1}},
\end{aligned}
$$

equals $\left.\left(X\left(\frac{d}{d t}\right) \ell_{1}(\cdot)\right)^{\top} \operatorname{col}\left(S \frac{d^{i}}{d t^{i}} M\left(\frac{d}{d t}\right) \ell_{2}(\cdot)\right)_{i=0, \ldots, L-1}\right|_{t_{0}} ^{t_{1}}$. In order to do so, apply Leibniz's rule for the differentiation of products to conclude that

$$
\begin{aligned}
& \sum_{k=1}^{L} \sum_{j=0}^{k-1}(-1)^{j} \ell_{1}(\cdot)^{(k-1-j) \top}\left(M_{k}^{\top} S M\left(\frac{d}{d t}\right) \ell_{2}(\cdot)\right)^{(j)} \\
& =\sum_{k=1}^{L} \sum_{j=0}^{k-1}(-1)^{j} \ell_{1}(\cdot)^{(k-1-j) \top} \sum_{i=0}^{j}\left(\begin{array}{l}
j \\
i
\end{array}\right) M_{k}^{(j-i) \top} S\left(M\left(\frac{d}{d t}\right) \ell_{2}(\cdot)\right)^{(i)} .
\end{aligned}
$$

In the last expression, observe that $\left(M\left(\frac{d}{d t}\right) \ell_{2}(\cdot)\right)^{(0)}$ is multiplied on the left by

$$
\sum_{k=1}^{L} \sum_{j=0}^{k-1}(-1)^{j} \ell_{1}(\cdot)^{(k-1-j) \top} M_{k}^{(j) \top} S=\left(\sum_{k=1}^{L} \sum_{j=0}^{k-1}(-1)^{j} M_{k}^{(j)} \frac{d^{(k-1-j)}}{d t^{(k-1-j)}} \ell_{1}(\cdot)\right)^{\top} S,
$$

$\left(M\left(\frac{d}{d t}\right) \ell_{2}(\cdot)\right)^{(1)}$ is multiplied on the left by

$$
\begin{aligned}
& \sum_{k=2}^{L} \sum_{j=1}^{k-1}(-1)^{j} \ell_{1}(\cdot)^{(k-1-j) \top}\left(\begin{array}{l}
j \\
1
\end{array}\right) M_{k}^{(j) \top} S \\
& =\left(\sum_{k=2}^{L} \sum_{j=1}^{k-1}(-1)^{j}\left(\begin{array}{c}
j \\
j-1
\end{array}\right) M_{k}^{(j)} \frac{d^{(k-1-j)}}{d t^{(k-1-j)}} \ell_{1}(\cdot)\right)^{\top} S,
\end{aligned}
$$


and so forth. These equalities, together with formula (4.1) for state trajectories for systems in image form, prove the claim of the lemma.

An argument symmetric to that used in the proof of Lemma 6.4 can be used to prove the following result.

Lemma 6.5. Let $M\left(\frac{d}{d t}\right)=M_{0}+M_{1} \frac{d}{d t}+\ldots+M_{L} \frac{d^{L}}{d t^{L}}$ be a polynomial differential operator with $q \times m$ meromorphic coefficients, and $S=S^{\top} \in \mathbb{R}^{q \times q}$. Define

$$
X\left(\frac{d}{d t}\right):=\operatorname{col}\left(\sum_{k=i+1}^{N} \sum_{j=i}^{k-1}(-1)^{j}\left(\begin{array}{c}
j \\
j-i
\end{array}\right) M_{k}^{(j-i)} \frac{d^{k-1-j}}{d t^{k-1-j}}\right)_{i=0, \ldots, L-1} .
$$

For every $\left[t_{0}, t_{1}\right] \subset \mathbb{R}$ such that $\left[t_{0}, t_{1}\right] \subseteq \operatorname{dom}\left(M_{k}\right), k=0, \ldots, L$, and every $\ell_{i}(\cdot) \in$ $\mathcal{C}^{\infty}\left(\left[t_{0}, t_{1}\right], \mathbb{R}^{m}\right), i=1,2$, it holds that

$$
\begin{aligned}
& \int_{t_{0}}^{t_{1}}\left(M\left(\frac{d}{d t}\right) \ell_{1}(\cdot)\right)^{\top} S\left(M\left(\frac{d}{d t}\right) \ell_{2}(\cdot)\right) d t \\
& =\left.\operatorname{col}\left(S \frac{d^{i}}{d t^{i}} M\left(\frac{d}{d t}\right) \ell_{1}(\cdot)\right)_{i=0, \ldots, L-1}^{\top}\left(X\left(\frac{d}{d t}\right) \ell_{2}(\cdot)\right)^{\top}\right|_{t_{0}} ^{t_{1}} \\
& +\int_{t_{0}}^{t_{1}}\left(M\left(\frac{d}{d t}\right)^{*} S M\left(\frac{d}{d t}\right) \ell_{1}(\cdot)\right)^{\top} \ell_{2}(\cdot) d t .
\end{aligned}
$$

We state a characterization of cyclo-losslessness.

Theorem 6.6. Let $\mathcal{B}=\operatorname{im} M\left(\frac{d}{d t}\right)$ and $S=S^{\top} \in \mathbb{R}^{q \times q}$. Define $X\left(\frac{d}{d t}\right)$ by (4.1). The following statements are equivalent:

1. $\mathcal{B}$ is cyclo-lossless with respect to the supply rate induced by $S$;

2. For every $\left[t_{0}, t_{1}\right] \subset \mathbb{R},\left[t_{0}, t_{1}\right] \subseteq \cap_{k=0}^{L} \operatorname{dom}\left(M_{k}\right)$, the polynomial differential operator

$$
\begin{aligned}
M\left(\frac{d}{d t}\right)^{*} S M\left(\frac{d}{d t}\right): \mathcal{C}^{\infty}\left(\left[t_{0}, t_{1}\right], \mathbb{R}^{m}\right) & \rightarrow \mathcal{C}^{\infty}\left(\left[t_{0}, t_{1}\right], \mathbb{R}^{m}\right) \\
\ell(\cdot) & \rightarrow M\left(\frac{d}{d t}\right)^{*} S M\left(\frac{d}{d t}\right) \ell(\cdot)
\end{aligned}
$$

is the zero operator, i.e. $M\left(\frac{d}{d t}\right)^{*} S M\left(\frac{d}{d t}\right) \ell(\cdot)=0 \forall \ell(\cdot) \in \mathcal{C}^{\infty}\left(\left[t_{0}, t_{1}\right], \mathbb{R}^{m}\right)$;

3. There exists a bilinear differential form $B_{\Psi}$ such that for every pair of functions $\ell_{i}(\cdot) \in \mathcal{C}^{\infty}\left(\left[t_{0}, t_{1}\right], \mathbb{R}^{m}\right), i=1,2$ it holds that

$$
\left(M\left(\frac{d}{d t}\right) \ell_{1}(\cdot)\right)^{\top} S\left(M\left(\frac{d}{d t}\right) \ell_{2}(\cdot)\right)=\frac{d}{d t} B_{\Psi}\left(\ell_{1}(\cdot), \ell_{2}(\cdot)\right) .
$$

4. There exists a bilinear differential form $B_{\Psi}$ such that for every $\left[t_{0}, t_{1}\right] \subset \mathbb{R}$ such that $\left[t_{0}, t_{1}\right] \subseteq \cap_{k=0}^{L} \operatorname{dom}\left(M_{k}\right)$,

$$
\left[\begin{array}{c}
M_{0}^{\top} \\
M_{1}^{\top} \\
\vdots \\
M_{L}^{\top} \\
0_{m \times q} \\
\vdots
\end{array}\right] S\left[\begin{array}{llllll}
M_{0} & M_{1} & \ldots & M_{L} & 0_{q \times m} & \ldots
\end{array}\right]=\frac{d}{d t} \widetilde{\Psi}+\sigma_{D}(\widetilde{\Psi})+\sigma_{R}(\widetilde{\Psi}) .
$$


Assume that any one of the statements 1.) - 4.) holds; then there exists $P \in \mathcal{M}^{q L \times q L}$ such that

$$
B_{\Psi}\left(\ell_{1}(\cdot), \ell_{2}(\cdot)\right)=\left(X\left(\frac{d}{d t}\right) \ell_{1}(\cdot)\right)^{\top} P\left(X\left(\frac{d}{d t}\right) \ell_{2}(\cdot)\right) .
$$

Proof. We first prove the equivalence of statements 1.)-4.).

The equivalence between statements 1.) and 2.) is a straightforward consequence of equation (6.7) in Lemma 6.4.

The equivalence of statements 2.) and 3.) follows from (6.7) and the fundamental theorem of integral calculus. For future reference, note that the bilinear differential form referred to in statement 3.) is

$$
B_{\Psi}\left(\ell_{1}(\cdot), \ell_{2}(\cdot)\right):=\left(X\left(\frac{d}{d t}\right) \ell_{1}(\cdot)\right)^{\top} \operatorname{col}\left(S \frac{d^{i}}{d t^{i}} M\left(\frac{d}{d t}\right) \ell_{2}(\cdot)\right)_{i=0, \ldots, L-1} .
$$

To prove the equivalence of statements 3.) and 4.), denote by $B_{\Phi}$ the bilinear differential form

$$
B_{\Phi}\left(\ell_{1}(\cdot), \ell_{2}(\cdot)\right):=\left(M\left(\frac{d}{d t}\right) \ell_{1}(\cdot)\right)^{\top} S\left(M\left(\frac{d}{d t}\right) \ell_{2}(\cdot)\right) ;
$$

then the $(i, j)$-th entry of $\widetilde{\Phi}$ equals $M_{i}^{\top} S M_{j}, i, j=0, \ldots$. Since bilinear differential forms and coefficient matrices are in bijective association with each other, the equality $B_{\Phi}=\frac{d}{d t} B_{\Psi}$ holds if and only if the equality $\widetilde{\Phi}=\frac{d}{d t} \widetilde{\Psi}+\sigma_{D}(\widetilde{\Psi})+\sigma_{R}(\widetilde{\Psi})$ also holds.

We now prove the second part of the claim. It follows from Lemmas 6.4 and 6.5 and the equivalence of statements 1.) and 2.) that for every $\left[t_{0}, t_{1}\right] \subseteq \operatorname{dom} \cap \cap_{k=0}^{L}\left(M_{k}\right)$, and for every $\ell_{i} \in \mathcal{C}^{\infty}\left(\left[t_{0}, t_{1}\right], \mathbb{R}^{m}\right)$ it holds that

$$
\begin{aligned}
& \int_{t_{0}}^{t_{1}}\left(M\left(\frac{d}{d t}\right) \ell_{1}(\cdot)\right)^{\top} S\left(M\left(\frac{d}{d t}\right) \ell_{2}(\cdot)\right) d t \\
& =\left.\operatorname{col}\left(S \frac{d^{i}}{d t^{i}} M\left(\frac{d}{d t}\right) \ell_{1}(\cdot)\right)_{i=0, \ldots, L-1}^{\top}\left(X\left(\frac{d}{d t}\right) \ell_{2}(\cdot)\right)^{\top}\right|_{t_{0}} ^{t_{1}} \\
& =\left.\left(X\left(\frac{d}{d t}\right) \ell_{1}(\cdot)\right)^{\top} \operatorname{col}\left(S \frac{d^{i}}{d t^{i}} M\left(\frac{d}{d t}\right) \ell_{2}(\cdot)\right)_{i=0, \ldots, L-1}\right|_{t_{0}} ^{t_{1}} .
\end{aligned}
$$

Consider the bilinear differential form $\mathcal{B}_{\Psi}$ defined in (6.9), and its coefficient matrix $\widetilde{\Psi}$. Denote by $\widetilde{X}$ the coefficient matrix of $X\left(\frac{d}{d t}\right)$, that is the infinite matrix of meromorphic functions (with only a finite number of nonzero entries) $\widetilde{X}$ defined by

$$
X\left(\frac{d}{d t}\right) \ell(\cdot)=\underbrace{\left[\begin{array}{llllll}
X_{0} & X_{1} & \ldots & X_{L} & 0_{q L \times m} & \ldots
\end{array}\right]}_{=: \widetilde{X}} \operatorname{jet}(\ell(\cdot)) .
$$

Denote by $\widetilde{F}$ the coefficient matrix associated with $\operatorname{col}\left(S \frac{d^{i}}{d t^{i}} M\left(\frac{d}{d t}\right)\right)_{i=0, \ldots, L-1}$, i.e.

$$
\widetilde{F} \operatorname{jet}(\ell(\cdot)):=\operatorname{col}\left(S \frac{d^{i}}{d t^{i}} M\left(\frac{d}{d t}\right) \ell(\cdot)\right)_{i=0, \ldots, L-1} .
$$

The coefficient matrix of $B_{\Psi}$ equals $\widetilde{\Phi}=\widetilde{X}^{\top} F$; from (6.10) it follows that

$$
B_{\Psi}\left(\ell_{1}(\cdot), \ell_{2}(\cdot)\right)=\operatorname{jet}\left(\ell_{1}(\cdot)\right)^{\top} \widetilde{X}^{\top} \widetilde{F} \operatorname{jet}\left(\ell_{2}\right)=\operatorname{jet}\left(\ell_{1}(\cdot)\right)^{\top} \widetilde{F}^{\top} \widetilde{X} \operatorname{jet}\left(\ell_{2}(\cdot)\right),
$$


holds for every $\ell_{i}(\cdot) \in \mathcal{C}^{\infty}\left(\left[t_{0}, t_{1}\right], \mathbb{R}^{m}\right), i=1,2$; conclude that $\widetilde{X}^{\top} \widetilde{F}=\widetilde{F}^{\top} \widetilde{X}$.

Using unimodular operations on $\widetilde{X}$ and $\widetilde{F}$, compute a factorization of $\widetilde{X}^{\top} \widetilde{F}$ of the form $\widetilde{X}^{\top} \widetilde{F}=\widetilde{X}^{\prime \top} G \widetilde{F}^{\prime}$, where $G$ is a nonsingular matrix with meromorphic entries and $\widetilde{X}^{\prime}, \widetilde{F}^{\prime}$ have full row rank. From the equality $\widetilde{X}^{\prime \top} G \widetilde{F}^{\prime}=\widetilde{F}^{\prime \top} G^{\top} \widetilde{X}^{\prime}$ conclude that the row space of $\widetilde{F}^{\prime}$ is contained in the row space of $\widetilde{X}^{\prime}$, and consequently in that of $\widetilde{X}$. The claim follows.

We now consider cyclo-dissipative systems represented in image form. The difference between the integral of the supply rate (6.1) and the quadratic storage function is the integral of a quadratic differential form, i.e. there exists a quadratic functional $Q_{\Delta}$ of $\ell$ and its derivatives such that

$$
\int Q_{\Phi}(\ell) d \tau-Q_{\Psi}(\ell)=\int Q_{\Delta}(\ell) d \tau
$$

The functional $Q_{\Delta}$ is called a dissipation rate. The dissipation equality (6.11) can be rewritten as $\int\left(Q_{\Phi}(\ell)-Q_{\Delta}(\ell)\right) d \tau=Q_{\Psi}(\ell)$, making evident that a system is cyclodissipative with respect to the supply rate $Q_{\Phi}$ if and only if it is cyclo-lossless with respect to the new supply rate $Q_{\Phi}-Q_{\Delta}$. The following result is a straightforward consequence of this observation.

Corollary 6.7. Let $\mathcal{B}=\operatorname{im} M\left(\frac{d}{d t}\right)$ and $S=S^{\top} \in \mathbb{R}^{q \times q}$. Define $X\left(\frac{d}{d t}\right)$ by (4.1). Assume that $\mathcal{B}$ is $S$-cyclo-dissipative, with a dissipation rate $Q_{\Delta}$ that is a quadratic function of $\ell$ and its derivatives. Then the storage function $Q_{\Psi}$ such that $\frac{d}{d t} Q_{\Psi}=Q_{\Phi}-Q_{\Delta}$ is a quadratic function of the state, i.e. there exists $P \in \mathcal{M}^{q L \times q L}$ such that

$$
Q_{\Psi}(\ell)=\left(X\left(\frac{d}{d t}\right) \ell\right)^{\top} P\left(X\left(\frac{d}{d t}\right) \ell\right) .
$$

REMARK 8. The second part of Th. 6.6 (equivalently, Cor. 6.7) has been proved in $[16,20]$ for linear, time-invariant systems and bilinear and quadratic functionals with constant coefficients. The argument there was based on the algebraic framework of one- and two-variable polynomial matrices representing such systems and functionals. Th. 6.6 is a generalization of that result to systems described by higher-order differential equations with time-varying coefficients. It is based on an argument that only uses the definition of state and straightforward linear algebra concepts. When applied to time-invariant systems and functionals, our proof uses a different technique to prove the same result as $[20,16]$.

7. Conclusions. Starting from an intrinsic, trajectory-based definition of state, we have provided a procedure to compute a state variable for systems described by higher-order differential equations with time-varying coefficients. We have shown that first-order representations of a system can be computed from such state variable, and that the storage function of a cyclo-lossless system can be written as a quadratic function of the state. Given the focus on state, our treatment of bilinear and quadratic functional of system variables and their derivatives was limited in scope to storage functions, and in methodology to working directly with differential operators. Algebraic techniques for non-commutative polynomial rings open up the possibility of developing a whole calculus of bilinear and quadratic differential forms with time-varying coefficients based on their representation by polynomial matrices with meromorphic coefficients, as was done in [20] for the case of functionals with constant coefficients. This line of research will be pursued elsewhere. 
Acknowledgment. The author would like to thank the Reviewers for their comments on previous versions of the manuscript, especially on issues related to dissipativity (see Rem. 7) and to the definition of state (see Rem. 1).

\section{REFERENCES}

[1] Çamlibel, M.K., Willems, J.C. and Belur, M.N., "On the dissipativity of uncontrollable systems", Proc. 42nd IEEE CDC, 2003.

[2] Cohn, P.M., Free Rings and Their Relations, Academic Press, London, 1971.

[3] Fröhler, S. and Oberst, U., "Continuous time-varying linear systems", Syst. Contr. Lett., vol. 35, pp. 97-110, 1998.

[4] Goos, J. and Pintelon, R., "Minimal state space realisation of continuous-time linear timevariant input-output models", Int. J. Control, vol. 89, no. 4, pp. 722-730, 2016.

[5] Hill, D.J. and Moylan, P.J., "Dissipative dynamical systems: basic input-output and state properties", J. Franklin Inst., vol. 309, no. 5, pp. 327-357, 1980.

[6] Hughes, T.H., "A theory of passive linear systems with no assumptions", Automatica, vol. 86, pp. 87-97, 2017.

[7] Ilchmann, A., Nürnberger, I. and Schmale, W., "Time-varying polynomial systems", Int. J. Contr., vol. 40, pp. 329-362, 1984.

[8] Ilchmann, A., "Time-varying linear systems and invariants of system equivalence", Int. J. Contr., vol. 42 , no. 4 , pp. $759-790,1985$.

[9] Ilchmann, A. and Mehrmann, V., "A behavioral approach to linear time-varying systems. Part 1: general theory", SIAM J. Contr. Opt., vol. 44, no. 5, pp. 1725-1747, 2005.

[10] Kotta, $\ddot{\mathrm{U}}$, and Tõnso, M., "Realization of discrete-time nonlinear input-output equations: Polynomial approach", Automatica, vol. 48, pp. 255-262, 2012.

[11] Kotta, Ü, and Tõnso, M., "Realisation of linear time-varying systems", Int. J. Control, vol. 90, no. 9, pp. 1951-1956, 2017.

[12] S. Lang, Real and Functional Analysis, third edition, Springer-Verlag, New York, 1993.

[13] Ore, O., "Theory of non-commutative polynomials", Ann. of Math., vol. 34, pp. 480-508, 1933.

[14] Pal, D. and Belur, M.N., "Dissipativity of uncontrollable systems, storage functions, and Lyapunov functions", SIAM J. Contr. Opt., vol. 47, no. 6, pp. 2930-2966, 2008.

[15] Rapisarda, P. and Willems, J.C., "State maps for linear systems", SIAM J. Contr. Opt., vol. 35, no. 3, pp. 1053-1091, 1997.

[16] Trentelman, H.L. and Willems, J.C., "Every storage function is a state function", Syst. Contr. Lett., vol. 32, pp. 249-260, 1997.

[17] van der Schaft, A.J. and Rapisarda, P., "State Maps from Integration by Parts", SIAM J. Contr. Opt.,vol. 49, no. 6, pp. 2415-2439, 2011.

[18] Willems, J.C. "Dissipative dynamical systems part I: General theory", Archive for Rational Mechanics and Analysis, vol. 45, no. 5, pp. 321-351, 1972.

[19] Willems, J.C. and Polderman, Introduction to Mathematical Systems Theory: A Behavioral Approach, Springer-Verlag, New York, 1998.

[20] Willems, J.C. and Trentelman, H.L. , "On quadratic differential forms", SIAM J. Contr. Optim., vol. 36, pp. 1703 - 1749, 1998.

[21] Willems, J.C., "The behavioral approach to open and interconnected systems", IEEE Contr. Syst. Mag., vol. 27, pp. 46-99, 2007.

[22] Wood, J. "Key problems in the extension of module-behaviour duality", Linear Algebra Appl., vol. 351-352, pp. 761-798, 2002.

[23] Zerz, E. "An algebraic analysis approach to linear time-varying systems", IMA J. Math. Contr. Inf., vol. 23, pp. 113-126, 2005.

[24] Zerz, E. "First order representations of time-varying linear systems", Proc. 2006 IEEE Conf. on Computer Aided Control Systems Design, Munich, Germany, vol. 23, pp. 113-126, 2006. 